\title{
Cramér-Rao lower bound for breakpoint distance estimation in a path-loss model
}

\author{
J. Manuel Castro-Arvizu ${ }^{\ddagger}$, Pau Closas ${ }^{\dagger}$, Juan A. Fernández-Rubio ${ }^{\ddagger}$ \\ $\ddagger$ Universitat Politècnica de Catalunya (UPC) Campus Nord, 08034 Barcelona (Spain) \\ ${ }^{\dagger}$ Centre Tecnològic de Telecomunicacions de Catalunya (CTTC) Av. Carl Friedrich Gauss 7, 08860 \\ Castelldefels, Barcelona (Spain)
}

\begin{abstract}
This paper addresses the problem of determining the Cramér-Rao lower bound (CRLB) for the parameters and breakpoint distance in a Path-Loss Channel model for Received Signal Strength (RSS) measurements. The path loss model is usually assumed for corrupted RSS measurements due to the shadow fading channel feature. In this paper the two-slope path loss model is considered, in which RSS measurements are modeled differently for close and far distances. Closed-form expressions for the CRLB parameters are derived for unknown breakpoint distance. For unknown parameters and breakpoint distance value, a Bayesian estimation method is proposed. The CRLB is then compared with the performance of the herein proposed method. The comparison illustrates convergence and efficiency of the Bayesian estimator.
\end{abstract}

\section{INTRODUCTION}

Indoor location positioning systems have become very popular in recent years. Due to the poor performance in indoor and urban areas by Global Navigation Satellite Systems (GNSS) features, new techniques for indoor location have been proposed. The growth in indoor applications in medical, industry, public safety, transport and logistics has focused the research in new techniques to face these needs.

There have been a multitude of emerging approaches for localization based on WLAN (IEEE 802.11x), UWB (IEEE 802.15.4), Zigbee, and other non-GNSS technologies. Wireless Indoor Positioning has become a trend because the inexpensive hardware and the already dense deployment of Access Points (APs) in urban areas. This work will focus on the signals of IEEE 802.11 wireless networks as the primary source of information to approach the localization problem.

For indoor environments, an alternative to estimate the distance between the user and the device (AP) is using the emitted signal strength. Signal attenuation methods attempt to calculate the signal path loss due to propagation. Theoretical and empirical models are used to translate the difference between the transmitted and received signal strength into a estimated range [1], [2]. Most of the network-based location estimations use RSS measurements.

This work is centered in WLAN RSS-based positioning systems. Although fingerprinting techniques are widely used [3], this paper is focused on geometric or statistical techniques [4] that are based on previous knowledge of the radio propagation channel model.

Propagation model can built the radio map and also account any changes in the environment. In order to evaluate the effectiveness of a given channel coding and processing technique before construction, a model of the channel that adequately describes the environment must be developed. Such analysis reduces the cost of developing a complex system by reducing the amount of hardware that has to be developed for performance evaluation.

There are several channel models in literature to characterize this channel [5], [6]. This work considers the IEEE 802.11x channel model to develop a novel calibration algorithm based on Bayesian statistics as well as to derive the theoretical accuracy bound of a method calibrating such a model. IEEE 802.11x channel model does not require an accurate floor plan of the building and can be implemented without using a third party software [7].

The paper is organized as follows. In Section II the RSS channel model is presented, which has the particularity of having two path loss regions depending on the distance to the transmitter. The theoretical estimation bound for such model is formulated in Section III and the estimation of the parameters of the path loss model is presented in Section IV following Bayesian approach. Section V shows computer simulations and Section VI concludes the paper.

\section{Two-Slope Path Loss Channel Model}

IEEE $802.11 \mathrm{x}$ channel models were originally based on the HiperLAN/2 channel models [2]. They were then updated to provide a better representation of indoor environments [8] such as small offices and residences providing six parameterizations of the model (termed A to F).

The multipath and shadowing fading present in indoor environments when the base-portable distance increases, the number of intervening obstacles and other reasons, make that path loss model do not always hold. Because this particular reason, the parameters employed in this model is site-specific [8] [9].

The path loss model considered in this work is summarized in this section. Path loss refers to the average loss in signal strength over distance. For indoor environments, the path loss depends on the relative distance between the AP and the sensing device [10]. For far distances $(5 \leq d \leq 30$ meters), path reflections from the environment (specially reflections from surrounding walls) generally result in a steeper overall drop in the signal strength at the receiver. 
The classical two-slope model assumes a linear dependence between the path loss (expressed in $\mathrm{dB}$ ) and the logarithm of the distance $d$ between the transmitter and the receiver [11]:

$$
L(d)=\left\{\begin{array}{cl}
S_{\alpha_{1}}(d) & \text { if } d \leq d_{b p} \\
S_{\alpha_{1}}\left(d_{b p}\right)+10 \alpha_{2} \log _{10}\left(d / d_{b p}\right) & \text { if } d>d_{b p}
\end{array}\right.
$$

where the first equation gives the path loss (in decibels) for close distances (distances less than $d_{b p}$, known as the breakpoint distance) and the second equation gives the path loss beyond $d_{b p}$. The $\alpha_{1}$ and $\alpha_{2}$ values are the slopes before and after $d_{b p}$, respectively. Also known as the path loss exponents.

The function $S_{\alpha_{1}}(d)$ was obtained by measurement campaigns using radio signal ray tracing methods, premeasured RSS contours centered in the receiver or multiple measurements at several base stations [12], [13], [14]. This expression applies to distances less than $d_{b p}$ and has a slope of $\alpha_{1}$. The expression for free space path loss $S(d)$ comes from the Friis equation with path loss $\alpha_{1}$ [12]:

$$
S_{\alpha_{1}}(d)=-10 \log _{10}\left[\frac{G_{t} G_{r} \lambda^{2}}{(4 \pi d)^{2}} \cdot \frac{1}{d^{\alpha_{1}}}\right],
$$

where $G_{t}, G_{r}$ are the transmitter and receiver antenna gains, respectively, $d$ is the distance between them in meters, and $\lambda$ is the wavelength of the transmitted carrier frequency. Assuming isotropic antennas, (2) becomes for $d \leq d_{b p}$,

$$
S_{\alpha_{1}}(d)=L_{o}+20 \log _{10}(d)+10 \alpha_{1} \log _{10}(d),
$$

and, for $d>d_{b p}$,

$$
S_{\alpha_{2}}(d)=S_{\alpha_{1}}\left(d_{b p}\right)+10\left(c_{2}+\alpha_{2}\right) \log _{10}\left(d / d_{b p}\right)
$$

where $L_{o}$ is a known constant and $c_{2}=3.5$ [8], [11].

For any given transmitter/receiver configuration, the regions surrounding these stations can differ, resulting in the received signal with strength differing from the nominal (1). This variation (known as shadow fading or log-normal shadowing) can be modeled by an additive zero-mean Gaussian random variable. The notation, $\chi_{\sigma^{2}} \sim \mathcal{N}\left(0, \sigma^{2}\right)$ is used.

Then, including shadow fading to (1), the model is

$$
L(d)= \begin{cases}S_{\alpha_{1}}(d)+\chi_{\sigma_{1}^{2}} & \text { if } d \leq d_{b p} \\ S_{\alpha_{2}}(d)+\chi_{\sigma_{2}^{2}} & \text { if } d>d_{b p}\end{cases}
$$

As happens for the path loss exponents, the variance values differ before and after the breakpoint distance. Typically the values depend on the scenario but in all cases it is observed that $\sigma_{1}^{2}<\sigma_{2}^{2}$ and $\alpha_{1}<\alpha_{2}$.

The standard deviation of the received power before and after breakpoint distance, $\sigma_{1}$ and $\sigma_{2}$, is expressed in units of $\mathrm{dB}$ and is assumed relatively constant with distance. Figure 1 represents a simulation of real measurements taken from the two-slope path loss model at different relative distances between the AP and the sensing device.

The goal in this work is to estimate the parameters of the model $\left(\alpha_{1}, \alpha_{2}, \sigma_{1}^{2}, \sigma_{2}^{2}, d_{b p}\right)$ based on raw RSS measurements. In Section III the theoretical lower bound is derived under the assumption of an unknown $d_{b p}$ which has not been addressed previously in the literature.

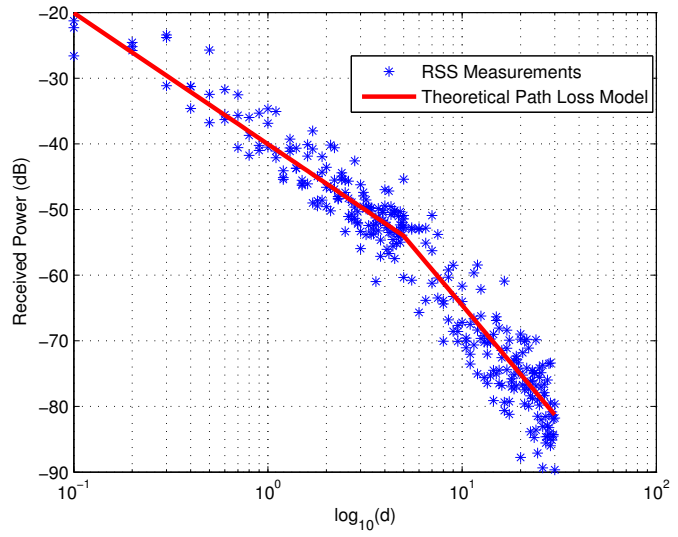

Fig. 1. Simulation of a two-slope path loss model with $\sigma_{1}=3 \mathrm{~dB}, \sigma_{2}=5 \mathrm{~dB}$, $\alpha_{1}=2, \alpha_{2}=3.5$, and $d_{b p}=5$ meters. Four RSS measurements per distance were recorded.

\section{CRAmÉr Rao Lower Bound}

This section derives closed-form expressions for the CRLB of the unknown parameters $\boldsymbol{\theta}=\left(\alpha_{1}, \sigma_{1}^{2}, \alpha_{2}, \sigma_{2}^{2}, d_{b p}\right)^{T}$. It is important to note that the CRLB derived in this section considers the case of unknown $d_{b p}$.

It is considered the typical case where $N$ independent observations are recorded at known distances to the AP under calibration, $\left\{d_{n}\right\}_{n=1}^{N}$ and $\boldsymbol{y}=\left\{y_{n} \triangleq L\left(d_{n}\right)\right\}_{n=1}^{N}$. The lower bounds are useful as a benchmark to judge estimation methods as well as to evaluate their consistency.

The likelihood function of the $N$ independent measurements for the two-slope model conditioned to the unknown $\boldsymbol{\theta}$ is:

$$
\begin{aligned}
p(\boldsymbol{y} \mid \boldsymbol{\theta}) & =p\left(y_{1}, y_{2}, \cdots, y_{N_{d_{b p}}} \mid \boldsymbol{\theta}\right) \\
& \cdot p\left(y_{N_{d_{b p}}+1}, y_{N_{d_{b p}}+2}, \cdots, y_{N} \mid \boldsymbol{\theta}\right)
\end{aligned}
$$

where $\left\{y_{1}, \cdots, y_{N_{d_{b p}}}\right\}$ are distributed according to the first model (before $d_{b p}$ ) and $\left\{y_{N_{d_{b p}}+1}, \cdots, y_{N}\right\}$ following the second model (after $d_{b p}$ ), as defined in (5).

Under the assumption that measurements are independent and Gaussian distributed we have that

$$
p(\boldsymbol{y} \mid \boldsymbol{\theta}) \sim \prod_{n=1}^{N_{d_{b p}}} \mathcal{N}\left(\mu_{1}(n), \sigma_{1}^{2}\right) \prod_{n=N_{d_{b p}}+1}^{N} \mathcal{N}\left(\mu_{2}(n), \sigma_{2}^{2}\right)
$$

where

$$
\begin{aligned}
\mu_{1}(n)= & L_{o}+20 \log _{10}(d)+10 \alpha_{1} \log _{10}(d) \\
\mu_{2}(n)= & L_{0}+20 \log _{10}\left(d_{b p}\right)+10 \alpha_{1} \log _{10}\left(d_{b p}\right)+ \\
& +10\left(c_{2}+\alpha_{2}\right) \log _{10}\left(d / d_{b p}\right) .
\end{aligned}
$$


Then, by definition,

$$
\begin{aligned}
p(\boldsymbol{y} \mid \boldsymbol{\theta})= & \prod_{n=1}^{N_{d_{b p}}} \frac{1}{\left(2 \pi \sigma_{1}^{2}\right)^{\frac{N_{d_{b p}}}{2}}} \exp \left[-\frac{\left(y_{n}-\mu_{1}(n)\right)^{2}}{2 \sigma_{1}^{2}}\right] \\
\cdot & \prod_{n=N_{d_{b p}}+1}^{N} \frac{1}{\left(2 \pi \sigma_{2}^{2}\right)^{\frac{N-N_{d_{b p}}}{2}}} \exp \left[-\frac{\left(y_{n}-\mu_{2}(n)\right)^{2}}{2 \sigma_{2}^{2}}\right]
\end{aligned}
$$

and applying natural logarithm to (9), we obtain

$$
\begin{aligned}
\ln p(\boldsymbol{y} \mid \boldsymbol{\theta}) & =-\frac{N_{d_{b p}}}{2}\left(\ln 2 \pi+\ln \sigma_{1}^{2}\right)- \\
& -\frac{N-N_{d_{b p}}}{2}\left(\ln 2 \pi+\ln \sigma_{2}^{2}\right)- \\
& -\frac{1}{2 \sigma_{1}^{2}} \sum_{n=1}^{N_{d_{b p}}}\left(y_{n}-\mu_{1}(n)\right)^{2}- \\
& -\frac{1}{2 \sigma_{2}^{2}} \sum_{n=N_{d_{b p}}+1}^{N}\left(y_{n}-\mu_{2}(n)\right)^{2}
\end{aligned}
$$

The $i, j$ element of the Fisher Information Matrix (FIM) is defined by,

$$
I_{i j}(\boldsymbol{\theta})=-\mathbb{E}\left\{\frac{\left.\partial^{2} \ln p(\boldsymbol{y} \mid \boldsymbol{\theta})\right)}{\partial \theta_{i} \partial \theta_{j}}\right\}
$$

Pluggin (10) in (11), the non-null FIM elements are calculated. This yields to:

$$
\begin{aligned}
& I_{11}=\frac{100}{\sigma_{1}^{2}} \sum_{n=1}^{N_{d_{b p}}}\left(\log _{10}\left(d_{n}\right)\right)^{2}+\frac{100\left(\log _{10}\left(d_{b p}\right)\right)^{2}}{\sigma_{2}^{2}}\left(N-N_{d_{b p}}\right) \\
& I_{22}=\frac{N_{d_{b p}}}{2 \sigma_{1}^{4}} \\
& I_{33}=\frac{100}{\sigma_{2}^{2}} \sum_{n=N_{d_{b p}}+1}^{N}\left(\log _{10} \frac{d_{n}}{d_{b p}}\right)^{2} \\
& I_{44}=\frac{N-N_{d_{b p}}}{2 \sigma_{2}^{4}} \\
& I_{55}=\frac{100\left(N-N_{d_{b p}}\right)\left(\alpha_{2}-\alpha_{1}\right)^{2}}{\sigma_{2}^{2} d_{b p}^{2}} \\
& I_{13}=I_{31}=\frac{100 \log _{10}\left(d_{b p}\right)}{\sigma_{2}^{2}} \sum_{n=N_{d_{b p}}+1}^{N} \log _{10} \frac{d_{n}}{d_{b p}} \\
& I_{51}=I_{15}=\frac{100\left(N-N_{d}\right)\left(2 \alpha_{1}-\alpha_{2}\right) \log _{10}\left(d_{b p}\right)}{\sigma_{2}^{2} d_{b p}} \\
& I_{53}=I_{35}=\frac{100\left(\alpha_{1}-2 \alpha_{2}\right)}{\sigma_{2}^{2} d_{b p}} \sum_{n=N_{d_{b p}}+1}^{N} \log _{10} \frac{d_{n}}{d_{b p}},
\end{aligned}
$$

and finally the FIM is formed as

$$
\mathbf{I}=\left(\begin{array}{ccccc}
I_{11} & 0 & I_{13} & 0 & I_{15} \\
0 & I_{22} & 0 & 0 & 0 \\
I_{31} & 0 & I_{33} & 0 & I_{35} \\
0 & 0 & 0 & I_{44} & 0 \\
I_{51} & 0 & I_{53} & 0 & I_{55}
\end{array}\right)
$$

The lower bound is obtained as the inverse of the FIM. This results in,

$$
\operatorname{CRLB}(\boldsymbol{\theta})=\left(\begin{array}{ccccc}
M_{11} & 0 & M_{13} & 0 & M_{15} \\
0 & \frac{1}{I_{22}} & 0 & 0 & 0 \\
M_{31} & 0 & M_{33} & 0 & M_{35} \\
0 & 0 & 0 & \frac{1}{I_{44}} & 0 \\
M_{51} & 0 & M_{52} & 0 & M_{55}
\end{array}\right)
$$

where,

$$
\begin{aligned}
M_{11} & =\frac{I_{11} I_{33} M_{C R L B}+\left[I_{15}\left(I_{11} I_{33}-I_{13}^{2}\right)-I_{13}\left(I_{11} I_{35}-I_{13} I_{15}\right)\right]^{2}}{I_{11}\left(I_{11} I_{33}-I_{13}^{2}\right) M_{C R L B}} \\
M_{33} & =\frac{I_{11}\left(I_{11} I_{55}-I_{15}^{2}\right)}{M_{C R L B}} \\
M_{55} & =\frac{I_{11}\left(I_{11} I_{33}-I_{13}^{2}\right)}{M_{C R L B}}
\end{aligned}
$$

and

$M_{C R L B}=\left(I_{11} I_{55}-I_{15}^{2}\right)\left(I_{11} I_{33}-I_{13}^{2}\right)-\left(I_{11} I_{35}-I_{13} I_{15}\right)^{2}$

The diagonal contains the values of the variance bounds of the parameter $\boldsymbol{\theta}$. The crossed values for $\alpha_{1}$ and $\alpha_{2}$ and with respect to $d_{b p}$ (elements $M_{13}, M_{15}$ and $M_{35}$ ), appear because each part of the two-slope path loss model depend on $\alpha_{1}$.

\section{MODEL PARAMETER ESTIMATION BY BAYESIAN INFERENCE}

This section addresses the design of an estimation method for $\boldsymbol{\theta}$. The statistical problem is to detect the change point in the means and variances of the RSS measurements, as well as estimating the other model parameters. The measurements follow the Gaussian distribution discussed in Section 2. Recall that the particularity is that mean and variance may change at one specific distance. Since the Bayesian approach is followed, an $a$ priori distribution for $\boldsymbol{\theta}$ has to be set. In this paper it is assumed a distribution that factorizes as:

$$
\boldsymbol{\theta} \sim \pi(\boldsymbol{\theta})=\pi\left(\alpha_{1}\right) \pi\left(\alpha_{2}\right) \pi\left(\sigma_{1}^{2}\right) \pi\left(\sigma_{2}^{2}\right) \pi\left(d_{b p}\right)
$$

An uniform prior over the full range of possible distances (defined as $d_{\max }$ ) is assumed for the $d_{b p}$, and conjugated priors are given to the path losses and the variances:

$$
\begin{aligned}
\alpha_{i} & \sim \pi\left(\alpha_{i}\right)=\mathcal{N}\left(0, \mathcal{V}_{\alpha_{i}}^{2}\right) \\
\sigma_{i}^{2} & \sim \pi\left(\sigma_{i}^{2}\right)=\Gamma^{-1}\left(a_{i}, b_{i}\right) \\
d_{b p} & \sim \pi\left(d_{b p}\right)=\mathcal{U}\left(0, d_{\max }\right)
\end{aligned}
$$

where $i=\{1,2\} . \mathcal{V}_{\alpha_{i}}^{2}, a_{i}$ and $b_{i}$ control the initial uncertainty on the parameters of the model. Since little knowledge is assumed, $\mathcal{V}_{\alpha_{i}}^{2}=0.0001, a_{i}=0.1$ and $b_{i}=0.0001$ values are used in the results section.

The problem of inferring the posterior distribution over the latent variables $\alpha_{1}, \sigma_{1}^{2}, \alpha_{2}$ and $\sigma_{2}^{2}$ could be solved analytically via Bayes theorem [15]. However, due to the unknown $d_{b p}$, computational methods such as Markov Chain Monte Carlo (MCMC) methods are needed. The MCMC algorithm combines the prior distribution with the likelihood to obtain the 
posterior distribution. MCMC algorithms are typically run for a large number of iterations (to achieve the convergence to the target posterior). Because samples from the early iterations are not from the target posterior, they are discarded. The discarded iterations is referred as the burn-in period [16].

The statistical model was implemented with Gibbs Sampling [17], which provides the joint posterior distribution of interest. To be implemented, the Gibbs sampler requires the posterior conditional for each of the latent variables. They are obtained, after applying basic probability theory, as

$$
\begin{aligned}
& \alpha_{1} \sim p\left(\alpha_{1} \mid \sigma_{1}^{2}, \alpha_{2}, \sigma_{2}^{2}, d_{b p}, \boldsymbol{y}\right)= \\
& \mathcal{N}\left(\frac{\mathcal{V}_{\alpha_{1}}^{2} \sum_{n=1}^{N_{d_{b p}}} y_{n}}{\sigma_{1}^{2}+\mathcal{V}_{\alpha_{1}}^{2} \cdot N_{d_{b p}}}, \frac{\sigma_{1}^{2} \cdot \mathcal{V}_{\alpha_{1}}^{2}}{\sigma_{1}^{2}+\mathcal{V}_{\alpha_{1}}^{2} \cdot N_{d_{b p}}}\right) \\
& \sigma_{1}^{2} \sim p\left(\sigma_{1}^{2} \mid \alpha_{1}, \alpha_{2}, \sigma_{2}^{2}, d_{b p}, \boldsymbol{y}\right)= \\
& \Gamma^{-1}\left(a_{1}+\frac{N_{d_{b p}}}{2}, b_{1}+\frac{\sum_{n=1}^{N_{d_{b p}}}\left(y_{n}-\mu_{1}(n)\right)}{2}\right) \\
& \alpha_{2} \sim p\left(\alpha_{2} \mid \alpha_{1}, \sigma_{1}^{2}, \sigma_{2}^{2}, d_{b p}, \boldsymbol{y}\right)= \\
& \mathcal{N}\left(\frac{\mathcal{V}_{\alpha_{2}}^{2} \sum_{n=N_{d_{b p}}+1}^{N} y_{n}}{\sigma_{2}^{2}+\mathcal{V}_{\alpha_{2}}^{2} \cdot\left(N-N_{d_{b p}}\right)}, \frac{\sigma_{2}^{2} \cdot \mathcal{V}_{\alpha_{2}}^{2}}{\sigma_{2}^{2}+\mathcal{V}_{\alpha_{2}}^{2} \cdot\left(N-N_{d_{b p}}\right)}\right) \\
& \sigma_{2}^{2} \sim p\left(\sigma_{2}^{2} \mid \alpha_{1}, \sigma_{1}^{2}, \alpha_{2}, d_{b p}, \boldsymbol{y}\right)= \\
& \Gamma^{-1}\left(a_{2}+\frac{N-N_{d_{b p}}}{2}, b_{2}+\frac{\sum_{n=N_{b_{b p}}+1}^{N}\left(y_{n}-\mu_{2}(n)\right)}{2}\right) \\
& d_{b p} \sim p\left(d_{b p} \mid \alpha_{1}, \sigma_{1}^{2}, \alpha_{2}, \sigma_{2}^{2}, \boldsymbol{y}\right) \propto p(\boldsymbol{y} \mid \boldsymbol{\theta}) \cdot \pi\left(d_{b p}\right),
\end{aligned}
$$

and thus the Bayesian solution based on Gibbs Sampling can be easily implemented.

\section{RESULts}

In this work the model IEEE 802.11c is used with the parameters set as in Figure 1. These values represent a typical office environment. The Gibbs sampler for the breakpoint distance model was implemented using WinBugs [18]. Two MCMC chains were simulated, each with $10^{4}$ Monte Carlo runs and 300 burn-in samples. The performance of the proposed algorithm was evaluated with 250 iterations to obtain the RMSE of each parameter in $\boldsymbol{\theta}$. These simulations illustrate the convergence shape of the RMSE according to its corresponding CRLB plot.

Two experiments were performed:

1 The experiment consisting in evaluating the effect of $N$ (number of samples) on $\hat{\boldsymbol{\theta}}$. In the simulation. The distance $d_{\max }=30$ meters was fixed and by adjusting $N$ (from 10 to 100) with one sample per distance, the granularity of RSS measurements in distance was adjusted.

$2 N$ was fixed in 10 and the amount of RSS measurements was modified by increasing from 1 to 15 samples per each distance.

The results of the first experiment are shown in Figure 2. The RMSE for every latent variable in comparative with their respective CRLB is plotted. The label CRLB $_{0}$ refers to the
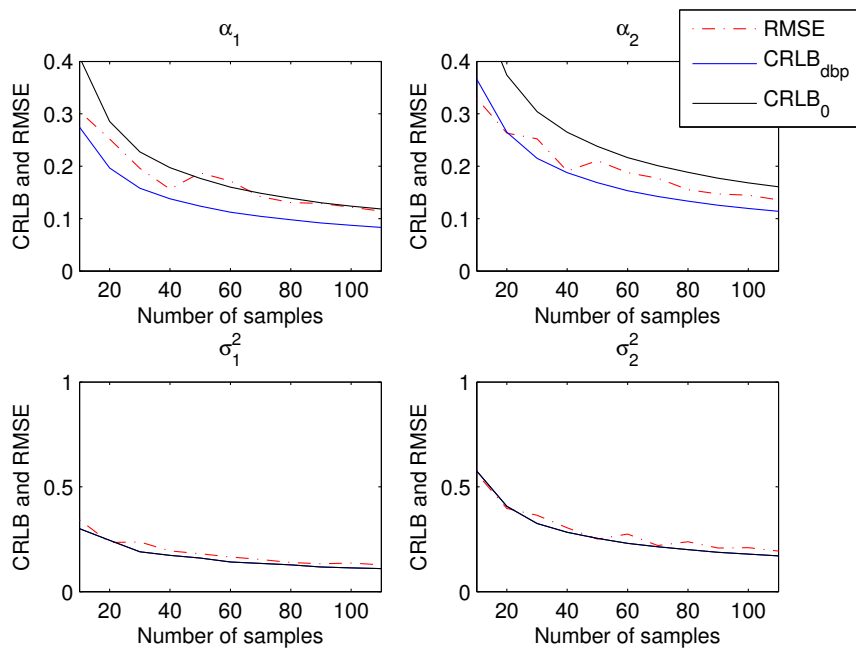

Fig. 2. Lower bounds of $\alpha_{1}, \sigma_{1}^{2}, \alpha_{2}, \sigma_{2}^{2}$, computed when the interval distance between each RSS measurements decreases in every iteration.
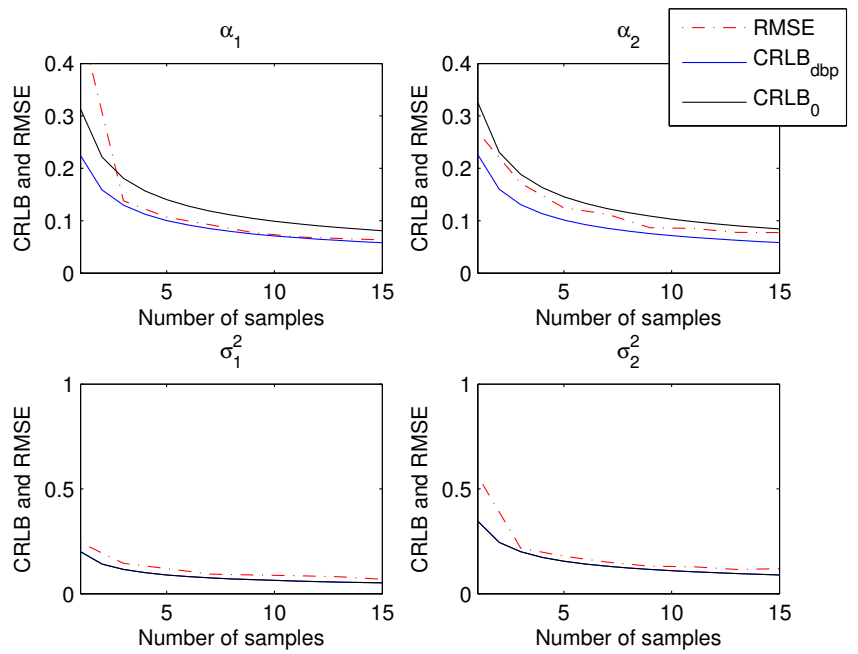

Fig. 3. Lower bounds of $\alpha_{1}, \sigma_{1}^{2}, \alpha_{2}, \sigma_{2}^{2}$, computed when the samples are increased per interval distance.

case when the parameters were estimated taking each part of the model separate and independently. $C R L B_{d b p}$ is the lower bound derived in this work.

It can be observed that the RMSE of the proposed algorithm attains the derived CRLB, regardless the assumptions of the bound are more optimistic when $d_{b p}$ is known. Figure 3 is for the second experiment with the same comparative as well as the first one.

According to every before established experiment, Figure 4 and 5 show the RMSE values for $d_{b p}$ and its corresponding lower bound.

These results show that the inference of $\boldsymbol{\theta}$ parameters achieve an asymptotically efficient performance. When RSS measurements samples are taken for fixed $N$, and number of samples per every interval distance are greater than one, the 


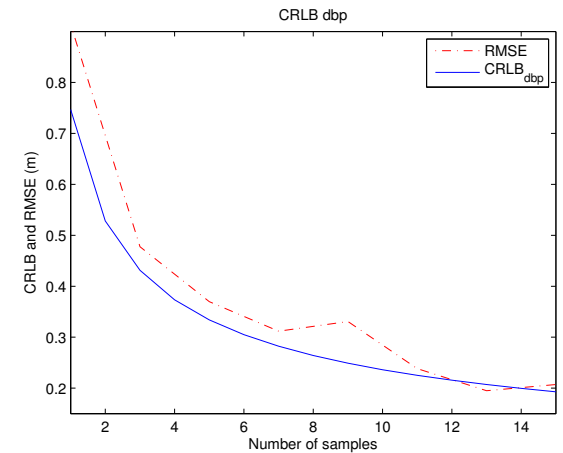

Fig. 4. Lower bound and RMSE of $d_{b p}$ when the samples are increased in every interval distance.

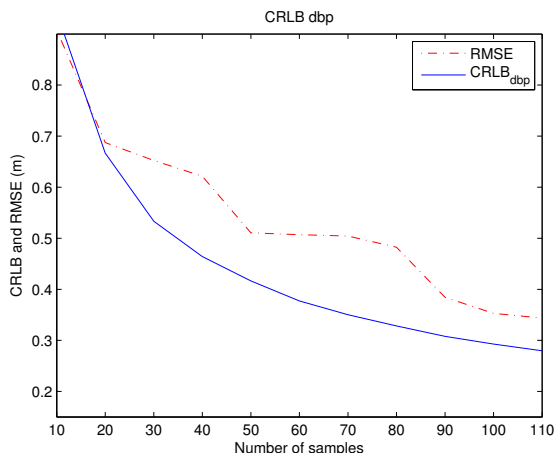

Fig. 5. Lower bound and RMSE of $d_{b p}$ when RSS measurements are taken when $N$ is decreased in every iteration.

results are promising.

\section{CONCLusions}

In this paper, a Bayesian approach for estimating the breakpoint distance, and the two-slope path loss model parameters in corrupted RSS measurements is presented. Additionally, this work proposes an implementation based on Gibbs sampling for which all the necessary posterior conditional distributions are shown. The CRLB for the case of unknown $d_{b p}$ is derived. The bound was compared with the evaluated RMSE of the proposed method, which estimates $d_{b p}$, The results are tight to the bound, thus indicates that the Bayesian method is estimating the five parameters in a consistent manner. Also, the joint estimation of the two-slope model parameters provides better results than individual fitting considering measurements from each of the models. Future work includes the evaluation of other a a priori distributions that could have better performance than the a a priori used in this work. Including the proposed methodology with real measurements is also envisaged.

\section{ACKNOWLEDGMENT}

This work has been partially supported by the Spanish Ministry of Economy and Competitiveness project TEC201239143 (SOSRAD), by the European Commission in the Net- work of Excellence in Wireless COMmunications NEWCOM $\sharp$ (contract n. 318306), by the Spanish Science and Technology Commission through project TEC2010-19171/TCM MOSAIC and by the National Council of Science and Technology (CONACyT) of Mexico.

\section{REFERENCES}

[1] H. Liu, H. Darabi, P. Banerjee, and J. Liu, "Survey of wireless indoor positioning techniques and systems," Systems, Man, and Cybernetics, Part C: Applications and Reviews, IEEE Transactions on, vol. 37, no. 6 , pp. 1067-1080, 2007.

[2] A. Hatami, "Application of channel modeling for indoor localization using TOA and RSS," PhD Dissertation, Worcester Polytechnic Institute, May 2006.

[3] K. El-Kafrawy, M. Youssef, A. El-Keyi, and A. Naguib, "Propagation Modeling for Accurate Indoor WLAN RSS-Based Localization," in Vehicular Technology Conference Fall (VTC 2010-Fall), 2010 IEEE 72nd, 2010, pp. 1-5.

[4] S. Gezici, "A Survey on Wireless Position Estimation," Wireless Personal Communications, vol. 44, no. 3, pp. 263-282, February 2008. [Online]. Available: http://dx.doi.org/10.1007/s11277-007-9375-z

[5] D. I. Laurenson, "Indoor radio channel propagation modelling by ray tracing techniques," PhD Dissertation, University of Edinburgh, 1994.

[6] T. Jamsa, T. Poutanen, and J. Meinila, "Implementation Techniques of Broadband Radio Channel Simulators," in Vehicular Technology Conference, 2001. VTC 2001 Spring. IEEE VTS 53rd, vol. 1, 2001, pp. 433-437 vol.1.

[7] M. Assad, M. Heidari, and K. Pahlavan, "Effects of Channel Modeling on Performance Evaluation of WiFi RFID Localization Using a Laboratory Testbed," in Global Telecommunications Conference, 2007. GLOBECOM '07. IEEE, 2007, pp. 366-370.

[8] L. S. V. Erceg, "TGn channel models. IEEE 802.11 Wireless LANs Document," IEEE 802.11-03/940r4, May, 2004.

[9] H. Hashemi, "The Indoor Radio Propagation Channel," Proceedings of the IEEE, vol. 81, no. 7, pp. 943-968, 1993.

[10] A. Sandeep, Y. Shreyas, S. Seth, R. Agarwal, and G. Sadashivappa, "Wireless Network Visualization and Indoor Empirical Propagation Model for a Campus Wi-Fi Network," World Academy of Science, Engineering and Technology, vol. 18, no. 6, pp. 705 - 710, 2008. [Online]. Available: http://waset.org/Publications? $\mathrm{p}=18$

[11] T. Paul and T. Ogunfunmi, "Wireless LAN Comes of Age: Understanding the IEEE 802.11n Amendment," Circuits and Systems Magazine, IEEE, vol. 8, no. 1, pp. 28-54, 2008.

[12] T. Rappaport, Wireless Communications: Principles and Practice, 2nd ed. Upper Saddle River, NJ, USA: Prentice Hall PTR, 2001.

[13] N. Patwari, A. Hero, M. Perkins, N. Correal, and R. O'Dea, "Relative Location Estimation in Wireless Sensor Networks," Signal Processing, IEEE Transactions on, vol. 51, no. 8, pp. 2137-2148, 2003.

[14] A. Coulson, A. Williamson, and R. Vaughan, "A Statistical Basis for Lognormal Shadowing Effects in Multipath Fading Channels," Cоттиnications, IEEE Transactions on, vol. 46, no. 4, pp. 494-502, 1998.

[15] J. Bernardo, Bayesian Statistics, University of Valencia, 46100, Burjassot, Valencia, Spain, November 2001.

[16] A. T. David Spiegelhalter, WinBUGS User Manual, 1st ed., Department of Epidemiology \& Public Health, Imperial College School of Medicine, Norfolk Place, London W2 1PG, UK, January 2003.

[17] A. F. M. Smith and G. O. Robert, "Bayesian computation via the Gibbs sampler and related Markov Chain Monte Carlo Methods," Journal of the Royal Statistical Society. Series B (Methodological), Vol. 55, No. 1, pp. 3-23, 1993.

[18] U. MRC Biostatistics Unit, Cambridge, "The BUGS Project. WinBUGS 1.4.3, http://www.mrc-bsu.cam.ac.uk/bugs/.” [Online]. Available: http://www.mrc-bsu.cam.ac.uk/bugs/ 